\title{
Evaluation of cytotoxic potential of cored soft contact lenses with adsorbed active ingredients from over-the-counter eye drops
}

\author{
Nobuhito Tabuchi' ${ }^{1}$ Manabu Hattori1, Makiko Shimizu², Misao Koide ${ }^{1}$ and Hiroshi Yamazaki ${ }^{2}$ \\ IPharmaceutical Research Laboratories No. 1, Research \& Development Headquarters, Lion Corporation, \\ 100 Tajima, Odawara, Kanagawa 256-0811, Japan \\ ${ }^{2}$ Laboratory of Drug Metabolism and Pharmacokinetics, Showa Pharmaceutical University, \\ 3-3165 Higashi-tamagawa Gakuen, Machida, Tokyo 194-8543, Japan
}

(Received January 6, 2012; Accepted January 27, 2012)

\begin{abstract}
Thin, gel-like, hydrated soft contact lenses are directly placed on human eyes and can be used with a variety of eye drops containing over-the-counter drugs, even though some labels suggest caution. In this study, evaluation was carried out of the cytotoxic potential of soft contact lenses that had adsorbed the active ingredients of over-the-counter eye drops. Although there are some guidelines for in vitro cytotoxicity tests for preclinical biological evaluation of medical materials and devices, the shape of soft contact lenses makes these tests difficult to apply. We developed a new cytotoxicity test in which cored lenses $6 \mathrm{~mm}$ in diameter were pretreated with active ingredients and placed in direct contact with Chinese hamster lung V79 cells. Among the 11 chemicals tested, anionic and hydrated type-IV lenses that had been soaked in benzalkonium chloride, berberine chloride, and chlorhexidine indicated cytotoxic potential toward the cultured cells. Solutions of $0.002 \%-0.004 \%$ benzalkonium chloride, $0.025 \%$ berberine chloride, and $0.008 \%$ chlorhexidine were not cytotoxic to the cultured cells under the experimental conditions; however, type-IV lenses that had been immersed in the same concentrations of these active ingredients showed significant cytotoxicity to the cultured cells. These results suggest that cytotoxicity to the ocular surface may be caused by soft contact lenses that have been soaked in the usual ranges of nontoxic eye drops containing benzalkonium chloride, berberine chloride, and chlorhexidine. This modified in vitro safety test system for cored soft contact lenses is of use for evaluating the cytotoxic potential of ophthalmological drugs adsorbed on soft contact lens surfaces.
\end{abstract}

Key words: Cytotoxicity, Soft contact lens, Active ingredients in eye drops, V79 cells, Benzalkonium chloride

\section{INTRODUCTION}

Thin, gel-like soft contact lenses are directly placed on eyes to correct various vision problems and tend to be compatible with modern lifestyles (Chalmers et al., 2011). However, some active components contained in ophthalmological drugs are unsuitable for use with soft contact lenses because of problems caused by the adsorption of the chemicals on soft contact lens surfaces. A previous clinical report showed that preservative-adsorbed soft contact lenses could cause diffuse superficial punctate keratopathy (Mizutani et al., 1995). Soft contact lenses are classified by the US Food and Drug Administra- tion into four groups based on their contents of ionic polymers and water (Tabuchi et al., 2009). We previously studied the mechanisms of adsorption of active ingredients in over-the-counter eye drops on soft contact lens surfaces (Tabuchi et al., 2009). The adsorption rates were increased with increasing hydrophobicity of the active ingredients; the adsorption was predominantly governed by hydrophobic interactions of active substances with soft contact lens surfaces, although electrostatic interactions played an additional role (Tabuchi et al., 2009). Two in vitro testing methods for the cytotoxicity of soft contact lenses with respect to Chinese hamster lung V79 cells are listed in guidelines for preclinical biological evaluation of

Correspondence: Nobuhito Tabuchi (E-mail: ntabuchi@lion.co.jp) 
medical materials and devices (MHLW, 2003; Tsuchiya, 1994). These cytotoxicity tests use the direct contact method and use extracts to determine the effect on colony formation. However, it should be pointed out that neither test system is particularly suitable for evaluating the effects of the uptake of drugs into actual lenses on eyes. Therefore, it is necessary to develop a new test system for evaluating the cytotoxicity of active ingredients in direct use with soft contact lenses.

As far as we know, however, the cytotoxicity of soft contact lenses soaked in over-the-counter ophthalmological drugs has not been characterized in detail. In the present study, we report evaluation of the cytotoxic potential of cored soft contact lenses $6 \mathrm{~mm}$ in diameter that have been soaked in active ingredients such as cationic benzalkonium chloride (used in over-the-counter eye drops) by direct contact with cultured cells.

\section{MATERIALS AND METHODS}

\section{Materials}

Three representative soft contact lenses, Precision UVTM $^{T}$, Bausch \& Lomb PureVision ${ }^{\circledR}$, and Acuvue ${ }^{\circledR}$, and active ingredients commercially available were purchased from the same sources as described previously (Tabuchi et al., 2009) unless otherwise specified; lenses were classified as type II $(<1 \mathrm{~mol} \%$ ionic polymers and $\geq 50 \%$ water), type III ( $\geq 1 \mathrm{~mol} \%$ ionic polymers and $<50 \%$ water), or type IV ( $\geq 1 \mathrm{~mol} \%$ ionic polymers and $\geq 50 \%$ water), corresponding to soft contact lens classification by the US Food and Drug Administration (Tabuchi et al., 2009). Cored lenses $6 \mathrm{~mm}$ in diameter were prepared for cytotoxicity tests by punching with an N-33 puncher (Kokuyo, Tokyo, Japan).

Test solutions containing each active ingredient were prepared using Palitzsch's buffer, consisting of $1.1656 \mathrm{~g}$ of boric acid, $0.1147 \mathrm{~g}$ of borax, and $0.31 \mathrm{~g}$ of sodium chloride in a total volume of $100 \mathrm{ml}$ (Momose, 1984) without any preservative chemicals; maximum concentrations of the test chemicals were set according to the standards for approval of the manufacture of over-the-counter drugs and the dictionary of drug additives. Six cationic ingredients $(0.025 \%$ berberine chloride hydrate, $0.03 \%$ chlorpheniramine maleate, $0.069 \%$ ketotifen fumarate (Wako Pure Chemical Industries, Osaka, Japan), 0.05\% tetrahydrozoline hydrochloride, $0.01 \%$ benzalkonium chloride (Maruishi Pharmaceutical, Osaka, Japan), and $0.008 \%$ chlorhexidine gluconate (Wako)), two anionic ingredients $(0.05 \%$ pranoprofen and $0.02 \%$ sodium gualenate hydrate), and three nonionic ingredients $(0.4 \%$ chlorobutanol (Merk, Tokyo,
Japan), $0.05 \%$ of $l$-menthol, and $0.02 \%$ propyl parahydroxybenzoate) were used (Tabuchi et al., 2009). For chlorhexidine gluconate and chlorobutanol, 1.0\% propylene glycol (ADEKA, Tokyo, Japan) was used as a solubilizer in the initial dissolution, and for $l$-menthol, $0.3 \%$ polysorbate 80 (Kao, Tokyo, Japan) was used.

\section{Cytotoxicity assay using cored lenses}

Chinese hamster lung V79 cells $\left(3 \times 10^{5 / \text { well, }}\right.$ JCRB0603, Health Science Research Resource Bank, Osaka, Japan) were seeded in a $24-w e l l$ plate with minimal essential medium containing $10 \%$ fetal bovine serum (Nacalai Tesque, Kyoto, Japan) and incubated at $37^{\circ} \mathrm{C}$ under $5 \% \mathrm{CO}_{2}$ in air for $48 \mathrm{hr}$. The cells were collected, washed with $100 \mu$ l of phosphate buffered saline, and suspended in $50 \mu \mathrm{l}$ of phosphate buffered saline. The test solution $(50 \mu \mathrm{l})$ or two cored lenses $6 \mathrm{~mm}$ in diameter that had been extensively soaked in $3 \mathrm{ml}$ of the test solution in a $10-\mathrm{ml}$ glass vial at $37^{\circ} \mathrm{C}$ for 7 days (Tabuchi et al., 2009) were added over the V79 cells (Fig. 1). To investigate the cytotoxicity, the cultured cells were incubated at $37^{\circ} \mathrm{C}$ under $5 \% \mathrm{CO}_{2}$ in air for $30 \mathrm{~min}$. Finally, the cells were harvested by trypsinization with $0.25 \%$ trypsin/ EDTA solution (GIBCO, Tokyo, Japan). After centrifugation and staining with $0.4 \%$ trypan blue (GIBCO), cells were counted manually under a phase contrast microscope. The viability of V79 cells was calculated as a percentage of the viability of control cells without test solutions or pretreated cored lenses. Statistical analysis was

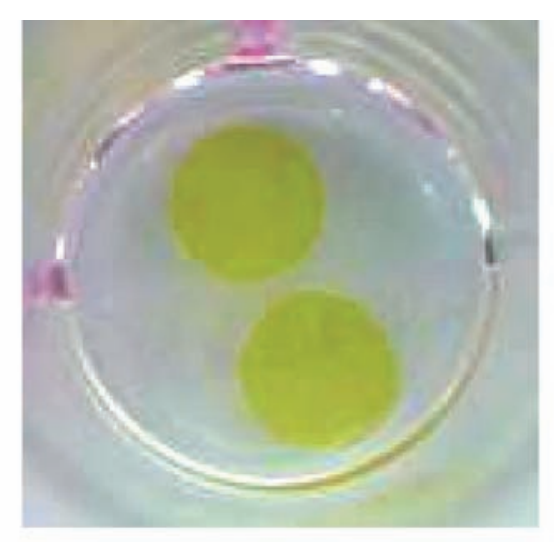

Fig. 1. A representative photograph for two cored lenses $6 \mathrm{~mm}$ in diameter that had been soaked in a test solution placed over the cells. Cored type IV lenses pretreated with berberine chloride $(0.025 \%)$ were used in this experiment. The apparent contact area of two cored lenses $6 \mathrm{~mm}$ in diameter with cells accounted for $33 \%$ of the entire area of each well. This experimental condition was selected because of good handling of both lenses and cells. 

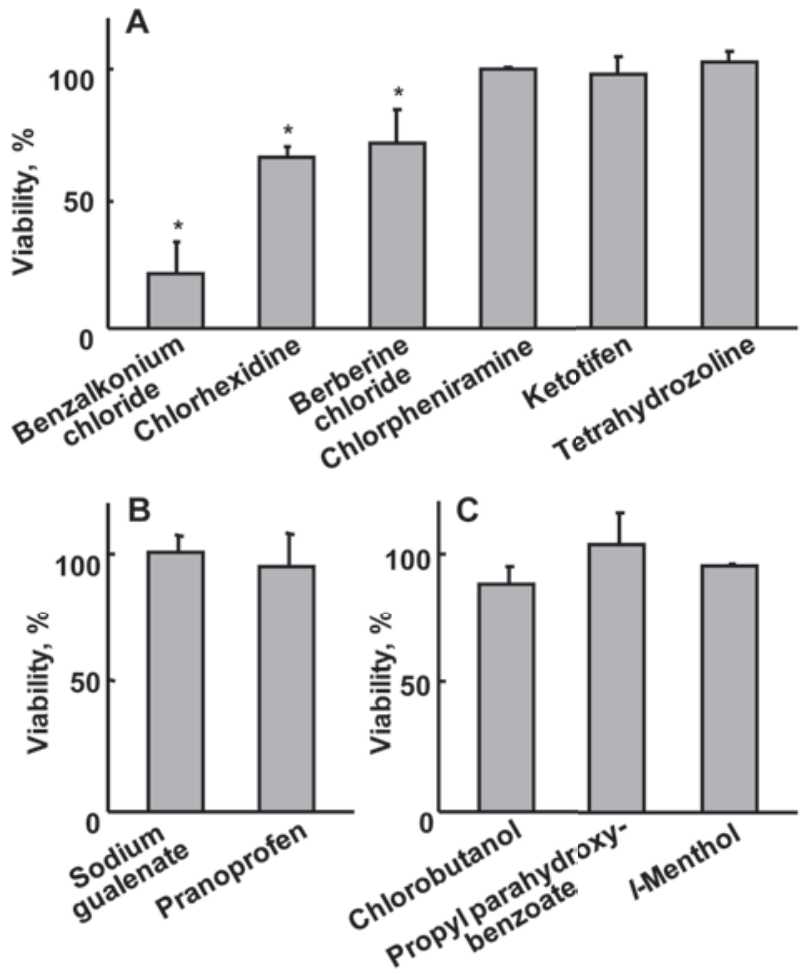

Fig. 2. Effects on cell viability of cored type-IV, -II, and -III lenses presoaked in solutions containing cationic (A), anionic (B), and nonionic (C) active ingredients in eye drops, respectively. Viable V79 cells stained with $0.4 \%$ trypan blue were manually counted under a phase microscope after two cored lenses $6 \mathrm{~mm}$ in diameter that had been soaked in an active-ingredient solution at $37^{\circ} \mathrm{C}$ for 7 days (Tabuchi et al., 2009) were placed over the cells for $30 \mathrm{~min}$ as described in Materials and methods. Results are expressed as mean values $\left( \pm\right.$ S.D.) of triplicate determinations $\left({ }^{*} p<0.05\right)$.

done using two-way analysis of variance (ANOVA) with Bonferroni post-tests or $t$ test (Prism, GraphPad Software, La Jolla, CA, USA).

\section{RESULTS AND DISCUSSION}

The viability of cultured V79 cells was investigated after direct contact with lenses presoaked in 1 of 11 cationic, anionic, or nonionic active-ingredient solutions. Cationic chlorpheniramine, ketotifen, and tetrahydrozoline did not affect the cell viability when adsorbed onto type-IV lenses under the current experimental conditions (Tabuchi et al., 2009) in which saturated adsorption was confirmed (Fig. 2A). Similarly, anionic sodium gualenate and pranoprofen (Fig. 2B) and nonionic chlo- robutanol, propyl parahydroxybenzoate, and $l$-menthol (Fig. 2C) did not affect the cell viability when adsorbed onto type-II and -III lenses, respectively, under conditions in which saturated adsorption was confirmed (Tabuchi et al., 2009). In contrast, type-IV lenses pretreated with benzalkonium chloride, chlorhexidine, or berberine chloride caused significant decreases in the cultured cell viability (Fig. 2A): adsorbed benzalkonium chloride resulted in $79 \%$ suppression of cell viability, compared with $34 \%$ suppression by chlorhexidine and $28 \%$ suppression by berberine chloride. In the present cytotoxicity study, the apparent contact area of the cored lens with cells accounted for $33 \%$ of the entire area of each well. Therefore, the more than $33 \%$ suppression mediated by benzalkonium chloride would suggest that adsorbed benzalkonium chloride exhibited cell toxicity by both direct and indirect actions on the biomarker cells.

Some studies have reported the adverse effects of benzalkonium chloride on the corneal epithelium (Gasset et al., 1974). A concentration range of $0.002 \%-0.005 \%$ benzalkonium chloride has been reported as less damaging to the ocular surface (Takahashi and Sasaki, 1989). We compared the cytotoxicities of $0.001 \%-0.01 \%$ benzalkonium chloride solutions alone and those of type-IV lenses immersed in solutions with the same concentration range of benzalkonium chloride. As shown in Fig. 3, the cultured cell viability for $0.001 \%-0.004 \%$ benzalkonium chloride solutions was approximately $100 \%$. On the other hand, when type-IV lenses were pretreated with $0.002 \%-0.004 \%$ benzalkonium chloride solutions, significant cytotoxic effects on the cultured cells were evident, as evaluated by two-way ANOVA (Fig. 3). In a preliminary study, benzalkonium chloride concentrations adsorbed on soft contact lenses were approximately 500 times higher than those in the corresponding solutions (not shown). Since it has been reported that 10\%-15\% by volume of adsorbed benzalkonium chloride may be released from lenses after $24 \mathrm{hr}$ immersion in $2 \mathrm{ml}$ saline (Ueda, 2007), relatively high concentrations of benzalkonium chloride seem to be released from type-IV samples and demonstrate higher cytotoxic potential than the corresponding solutions alone.

Similarly, we evaluated the adsorbed toxicity of $0.008 \%$ chlorhexidine and $0.025 \%$ berberine chloride solutions. As shown in Fig. 4, these sample solutions were not cytotoxic; however, type-IV lenses soaked in these chlorhexidine and berberine chloride solutions were significantly cytotoxic. Chlorhexidine solution (0.001\%) has been shown to be cytotoxic to V79 cells after $6 \mathrm{hr}$ of incubation (Kimura, 1985). The cytotoxicity of berberine chloride with respect to V79 cells has not been reported 


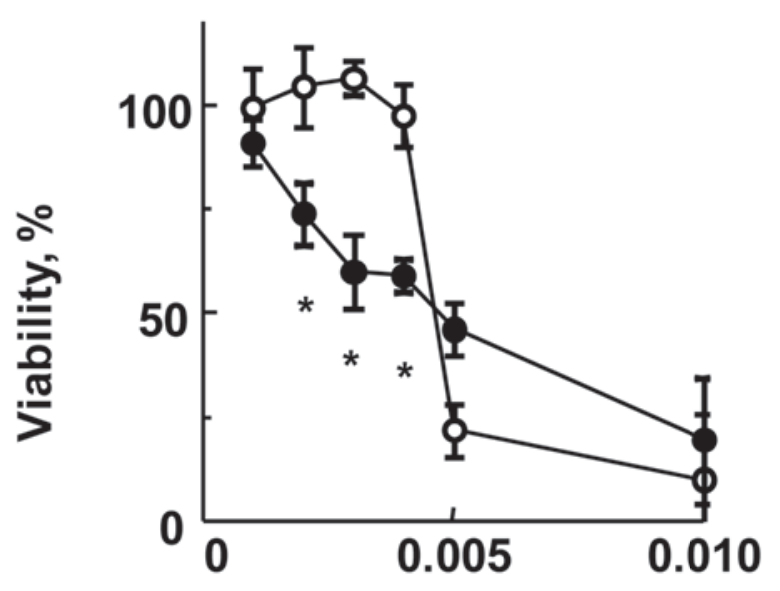

[Benzalkonium chloride], \%

Fig. 3. Effects on cell viability of cored type-IV lenses presoaked in benzalkonium chloride solutions when placed over cells for $30 \mathrm{~min}$. Two cored lenses $6 \mathrm{~mm}$ in diameter were soaked in $0.001 \%-0.01 \%$ benzalkonium chloride solutions at $37^{\circ} \mathrm{C}$ for 7 days (Tabuchi et al., 2009) and then placed over the cells (•). Corresponding benzalkonium chloride solutions ( $(0)$ were added to cells as controls. Details are given in Materials and methods. Results are expressed as mean values $\left( \pm\right.$ S.D.) of triplicate determinations $\left({ }^{*} p<0.01\right.$, two-way ANOVA with Bonferroni post-tests).

yet, although berberine chloride has been shown to have a wide range of pharmacological effects against human cancer cells (Hano, 1957), human leukemia HL-60 cells, and mice leukemia WEHI-3 cells (Lin et al., 2006; Yu et $a l ., 2007)$. We hypothesized that the cytotoxicity of berberine chloride and chlorhexidine adsorbed on type-IV lenses should be higher than that of each solution because of the confirmed high concentrations of chlorhexidine and berberine chloride adsorbed on lenses in our preliminary study (data not shown).

The present results show that when noncytotoxic ophthalmological drugs that include ingredients such as benzalkonium chloride, chlorhexidine, or berberine chloride are used in conjunction with soft contact lenses, adsorbed and accumulated ingredients may cause unexpected cytotoxicity to the human ocular surface. The present new in vitro test system for cultured cell cytotoxicity is of use for understanding the cytotoxic potential of ophthalmological drugs when used with soft contact lenses. Generally, $7 \mu \mathrm{l}$ of tear fluid is present on human eyes at a given time.

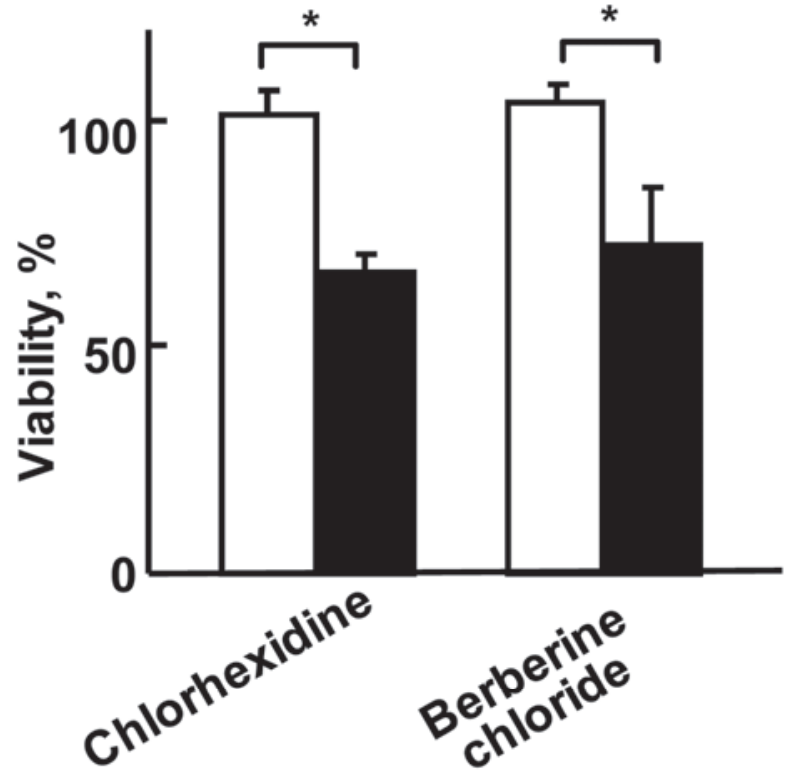

Fig. 4. Effects on cell viability of cored type-IV lenses presoaked in chlorhexidine or berberine chloride solutions when placed over cells for $30 \mathrm{~min}$. Two cored lenses $6 \mathrm{~mm}$ in diameter were soaked in $0.008 \%$ chlorhexidine or $0.025 \%$ berberine chloride solutions at $37^{\circ} \mathrm{C}$ for 7 days (Tabuchi et al., 2009) and then placed over cells for $30 \mathrm{~min}(\mathbf{\square})$. Berberine chloride and chlorhexidine solutions $(\square)$ were added to cells as controls. Details are given in Materials and methods. Results are expressed as mean values ( \pm S.D.) of triplicate determinations $(* p<0.05)$.

Eyes have been reported to produce $1.2 \mu \mathrm{l}$ of tear fluid per minute, which means that all tear fluid on the eyes is replaced roughly every 5 min (Honda, 1999). Therefore, when evaluating the cytotoxicity of ophthalmological drugs used in conjunction with soft contact lenses, it is necessary to consider not only the adsorption of active ingredients but also their desorption based on the amount of tear fluid present on the eyes and the rates of replenishment of tears. In the future, correlation studies will be necessary between the in vivo rabbit eye irritancy test (Tsuchiya et al., 1993) and this new test system for in vitro cytotoxicity of soft contact lenses.

In conclusion, in the present study we developed a modified evaluation method using cored lenses to evaluate the cytotoxicity of active ingredients adsorbed on soft contact lenses. The toxicological potential of benzalkonium chloride, chlorhexidine, and berberine chloride should be considered if they are directly applied onto human eyes when soft contact lenses are being worn. 
Cytotoxicity of actives-saturated soft contact lenses

\section{REFERENCES}

Chalmers, R.L., Wagner, H., Mitchell, G.L., Lam, D.Y., Kinoshita, B.T., Jansen, M.E., Richdale, K., Sorbara, L. and McMahon, T.T. (2011) : Age and other risk factors for corneal infiltrative and inflammatory events in young soft contact lens wearers from the Contact Lens Assessment in Youth (CLAY) study. Invest. Ophthalmol. Vis. Sci., 52, 6690-6696.

Gasset, A.R., Ishii, Y., Kaufman, H.E., and Miller, T. (1974): Cytotoxicity of ophthalmic preservatives. Am. J. Ophthalmol., 78, 98-105.

Hano, K. (1957): Pharmacological studies on metabolism of cancer tissues: pharmacological studies on carcinostatic effects of some plant components and their derivatives (1). Gann, 48, 443-455.

Honda, Y. (1999): Ganka Sinryo Practice, pp.92-93, Bunkodo Co., Tokyo.

Kimura, K. (1985): Effect of chlorhexidine on cultured V79 cells. Shigaku., 73, 378-389.

Lin, C.C., Kao, S.T., Chen, G.W., Ho, H.C. and Chung, J.G. (2006): Apoptosis of human leukemia HL-60 cells and murine leukemia WEHI-3 cells induced by berberine through the activation of caspase-3. Anticancer. Res., 26, 227-242.

MHLW (Ministry of Health, Labour and Welfare, Japan) (2003): Guideline for preclinical biological evaluation of medical materials and devices. MHLW Notice No. 36.

Mizutani, S., Ito, Y., Nishida, S., Mizutani, Y., Ito, T., Kono, M., and Mizutani, J. (1995): A spectrophotometric study of extraneous matter on contact lenses. J. Jpn. Contact Lens. Soc., 37, 154157.

Momose, K. (1984): Eye drop, pp.66-67, 171, Nanzando, Tokyo, Japan.

Tabuchi, N., Watanabe, T., Hattori, M., Sakai, K., Sakai, H. and Abe, M. (2009): Adsorption of actives in ophthalmological drugs for over-the-counter on soft contact lens surfaces. J. Oleo Sci., 58, 43-52.

Takahashi, N. and Sasaki, K. (1989): Preservatives and effect of preservatives on eyes. Ganka., 31, 43-48.

Tsuchiya, T. (1994): Studies on the standardization of cytotoxicity tests and new standard reference materials useful for evaluating the safety of biomaterials. J. Biomater. Appl., 9, 138-157.

Tsuchiya, T., Arai, T., Ohhashi, J., Imai, K., Kojima, H., Miyamoto, S., Hata, H., Ikarashi, Y., Toyoda, K., Takahashi, M., and Nakamura, A. (1993): Rabbit eye irritation caused by wearing toxic contact lenses and their cytotoxicities: In vivo/in vitro correlation study using standard reference materials. J. Biomed. Mater. Res., 27, 885-893.

Ueda, K. (2007): Adsorption and release of preservative from soft contact lenses. J. Jpn. Contact Lens Soc., 49, 181-186.

Yu F.S., Yang J.S., Lin H.J., Yu C.S., Tan T.W., Lin Y.T., Lin C.C., Lu H.F. and Chung J.G. (2007): Berberine inhibits WEHI-3 leukemia cells in vivo. In Vivo, 21, 407-412. 